\title{
Meeting the challenge of long COVID
}

\author{
In formulating the response of the healthcare system to the COVID-19 pandemic, the true toll of the chronic \\ consequences of SARS-CoV-2 infection must be uncovered and strategies must be devised for providing integrated \\ care to those with long-term illness.
}

T he health impact of the COVID-19 pandemic has typically been tracked through reporting of the number of cases, hospitalizations and deaths. But death is not the only important negative consequence of SARS-CoV-2 infection. It has become increasingly clear that many patients, even those with mild cases, may go on to develop lasting symptoms that can have disabling consequences for those affected. These patients, the so-called 'long haulers', report a wide variety of symptoms-extreme fatigue, muscle and joint pain, breathlessness, heart palpitations, loss or alteration of taste and smell, gastrointestinal distress, and problems with attention, memory and cognition. Although considerable progress has been made in treating the acute phase of SARS-CoV-2 infection, very little is known about the chronic impact of infection, which has come to be called 'long COVID'.

At this early stage in our understanding of long COVID, basic information on its true burden is urgently needed, including data on the incidence of long COVID in males relative to that in females, in different age and ethnic groups, and in relation to existing co-morbidities. This effort will require systematic follow-up with all patients diagnosed with COVID-19, for which digital monitoring using mobile devices might be well suited. There must also be agreed-upon diagnostic criteria for long COVID and a way to 'tag' people as having long COVID in their electronic health records, which will allow aggregation and analysis of data.

Estimates of the number of people with long COVID vary widely. In July, the US Centers for Disease Control and Prevention reported that one in five patients 18-34 years of age without chronic medical conditions and with a positive outpatient test had not fully recovered by $2-3$ weeks after testing, and a research center in Rome found that $87 \%$ of 143 patients reported persistence of at least one symptom 60 days after onset of the disease. According to a preprint published in October that has not yet undergone peer review, $24 \%$ of 233 patients still had symptoms at 90 days after infection. Data from a smartphone app, as reported by the COVID Symptom Study, showed that one in ten patients with COVID-19 have symptoms after 3 weeks. Given the scale of the pandemic, if even only a small percentage of the tens of millions of infected people worldwide develop long COVID, a staggeringly large number of people would need long-term follow-up and treatment.

Many different types of viruses can cause long-term illness, and from this perspective, the phenomenon of long COVID is hardly surprising. For example, infectious mononucleosis caused by Epstein-Barr virus can lead to persistent symptoms, and Guillain-Barré syndrome is a chronic neurological condition that can arise after viral infection. But what sets the SARS-CoV-2 virus apart is the sheer number of infected people and the damaging effects of infection on multiple organ systems, including the lungs, liver, brain, kidneys and heart. Research is urgently needed to delineate how SARS-CoV-2 infection can trigger this type of multifaceted syndrome. It remains unclear if the virus persists in patients with long COVID and whether chronic symptoms are due to the direct results of SARS-CoV-2 infection in multiple organs or to indirect effects, such as hyperactivation of the immune system or the development of autoimmunity.

The remarkable diversity of symptoms in patients with long COVID underscores the need to understand how symptoms evolve in individual patients and how this relates to their health outcomes. Another piece of the puzzle is understanding who is at risk of developing long COVID-for example, how disease severity and symptoms in the acute phase and the presence of co-morbid conditions might be related to longer-term consequences, and whether the immune response of people who develop long
COVID differs from that of those who do not. It will also be important to study whether there are ways of managing the acute disease that lower the risk of long-term consequences. For effective treatment of long COVID, the healthcare system will need to embrace new technologies and strategies, including telehealth and mobile health. The integration of digital technologies into healthcare must take into account the need to reach into historically underserved populations, particularly given the high burden of COVID-19 in such communities.

Care for patients with long COVID also needs to grapple with the reality that these patients can have a wide range of symptoms that would typically require the involvement of multiple types of specialists, which can result in a logistical nightmare for the patient seeking diagnosis and treatment. A more integrated approach is needed to ease the burden placed on the patient and improve care. Centers for the treatment of patients with long COVID that provide care spanning multiple medical specialties are now springing up, such as the Center for Post-COVID Care, at Mount Sinai hospital in New York City, and the Post-COVID Assessment and Recovery Clinic at Penn Medicine. Most recently, on 15 November 2020, the UK's National Health Service announced the launch of 40 clinics for the treatment of long COVID.

With highly effective vaccines against COVID-19 on the horizon, it is tempting to think that the pandemic will soon be resolved. But only with an understanding of the prevalence of long COVID and the chronic health consequences of SARS-CoV-2 infection will it be possible to determine the true impact of the pandemic and to adapt healthcare to effectively diagnose, monitor and treat patients over the long term.

Published online: 7 December 2020 https://doi.org/10.1038/s41591-020-01177-6 